SHORT REPORT

\title{
Increased systemic levels of norsalsolinol derivatives are induced by levodopa treatment and do not represent biological markers of Parkinson's disease
}

\author{
J Scholz, I Klingemann, A Moser
}

J Neurol Neurosurg Psychiatry 2004;75:634-636. doi: 10.1136/jnnp.2003.010769

Endogenously synthesised norsalsolinol derivatives are elevated in Parkinson's disease (PD) and have been considered potentially useful biological markers of the disease. However, little is known about the impact of dopaminergic drugs on the formation of these compounds. We prospectively examined the urine concentrations of norsalsolinol, $\mathrm{N}$-methyl-norsalsolinol and salsolinol in 47 PD patients and 14 control subjects. Patients and control subjects were re-examined after approximately 1 year to assess long term changes. Norsalsolinol derivatives were low in controls and untreated patients with early PD. Increased urine concentrations of norsalsolinol derivatives were significantly associated with levodopa treatment. They were elevated more markedly in the urine of patients treated with high (>600 mg daily) doses of levodopa compared with patients receiving medium (300$600 \mathrm{mg}$ ) or low ( $<300 \mathrm{mg}$ ) doses of the drug. There was no correlation with disease parameters such as the severity of motor disability or deficits in the cognitive performance. In the patient group, the concentrations of all three norsalsolinol derivatives declined over the period of investigation, however, they still remained elevated compared with the control group. We conclude that systemic levels of norsalsolinol derivatives in treated patients with PD are likely to derive from the metabolism of levodopa and cannot be regarded as intrinsic markers of the disease. The limited ability of norsalsolinol derivatives to pass the blood-brain barrier prevents an intracerebral accumulation of these possibly harmful compounds, which are biochemically similar to the neurotoxin 1-methyl-4-phenyl-1,2,3,6-tetrahydropyridine.

$\mathrm{N}$ orsalsolinol derivatives are 6,7-dihydroxylated tetrahydroisoquinolines, endogenously formed from dopamine and aldehydes. ${ }^{1}$ Elevated levels of norsalsolinol (Norsal), $N$-methyl-norsalsolinol (NMnorsal), and salsolinol have been found in the brain, ${ }^{2}$ the cerebrospinal fluid $(\mathrm{CSF})^{3-6}$ and the urine $e^{7}$ of patients with idiopathic Parkinson's disease (PD). Norsal derivatives have been proposed as biological markers of PD as they may result from an altered metabolism of dopamine in these patients. ${ }^{5}$ They are absent or found at low concentrations in healthy control subjects ${ }^{255}$ or in patients with motor disorders caused by multiple system atrophy. ${ }^{6}$ Biological markers would be valuable for the diagnosis of early PD and for monitoring the effects of neuroprotective or restorative treatment strategies against PD. Systemic markers would be readily accessible and allow repetitive measurements as opposed to examinations of the CSF. On the other hand, investigators who did not detect increased systemic or CSF levels of salsolinol in untreated patients with early $\mathrm{PD}$, argued that the presence of 6,7-dihydroxylated tetrahydroisoquinolines might be a consequence of therapeutically administered levodopa (L-dopa), which is subsequently decarboxylated to dopamine..$^{8-10}$ However, this issue has not been studied directly.

We prospectively measured urine levels of Norsal, NMnorsal, and salsolinol in PD patients and control subjects. Individuals were examined again after approximately one year. Our objective was to determine whether systemic concentrations of Norsal derivatives represent useful biological markers of PD, how they change over time in the individual patient, and whether they are affected by drug treatment.

\section{PATIENTS AND METHODS \\ Patients}

Eighteen women and 29 men (mean (SD) age 73.0 (10.1) years) with probable $\mathrm{PD}^{11}$ were enrolled. The mean (SD) duration of the disease was 9.2 (4.9) years. Patients with other neurological disorders, systemic metabolic diseases, or a history of alcoholism ${ }^{12}$ were excluded. The control group consisted of seven women and seven men of comparable age (70.8 (10.3) years) without neurodegenerative or metabolic disorders, or a history of alcohol abuse.

Patients were examined upon entry into the study (Tl) and 303.6 (111.4) days thereafter (T2). In the control group, the time interval between T1 and T2 was 360 (40.2) days. Urine was collected over 12 hours, including the first urine of the morning prior to drug intake. Study participants were not allowed to eat food rich in Norsal derivatives or to drink alcoholic beverages for 48 hours before urine samples were obtained. ${ }^{13}$ The severity of the parkinsonian syndrome was evaluated using the motor section of the Unified Parkinson's Disease Rating Scale (UPDRS). ${ }^{14}$ Cognitive impairment was assessed by the Mini-Mental State Examination (MMSE). ${ }^{15}$ Treatment with L-dopa and additional drugs including selegiline, inhibitors of catechol $O$-methyltransferase and dopamine agonists was documented. Three patients with early PD were untreated.

The study was approved by the ethics committee of the Medical University of Lübeck. Informed consent of patients and control subjects was obtained.

\section{Urine analysis}

Urine was collected in the presence of semicarbazide and $\mathrm{Na}_{2}$-EDTA. After centrifugation at $200 \mathrm{~g}$ for $10 \mathrm{~min}$, the supernatant was incubated in acetate buffer $(\mathrm{pH} 5.0), 5 \mathrm{U}$

\footnotetext{
Abbreviations: CSF, cerebrospinal fluid; MMSE, Mini Mental State Examination; NMNorsal, N-methyl-norsalsolinol; Norsal, norsalsolinol; PD, Parkinson's disease; UPDRS, Unified Parkinson's Disease Rating Scale
} 
arylsulfatase IV and $350 \mathrm{U} \beta$-glucuronidase (Sigma) at $30^{\circ} \mathrm{C}$ overnight. Subsequently, $\mathrm{pH}$ was brought to 8.6 and aliquots were loaded onto an $m$-amino-phenylboronic acid agarose cartridge. Norsal derivatives were eluted with acetic acid and quantified by high performance liquid chromatography with electrochemical detection as previously described, ${ }^{7}$ using a C18 column (Eurospher RP 18, particle size $5 \mu \mathrm{m}$, column size $250 \times 4.0 \mathrm{~mm}$ ) and a precolumn (column size $35 \times 4.0 \mathrm{~mm}$ ). 3,4-Dihydroxybenzylamine was used as internal standard. The detection limit of all three Norsal derivatives was $5 \mathrm{pmol} / \mathrm{ml}$. Values were corrected for a urine volume of $1000 \mathrm{ml}$.

\section{Statistics}

Statistical evaluations were conducted using the Statistical Package for the Social Sciences (SPSS, version 9.0). A two tailed Mann-Whitney $U$ test was applied for comparisons between patients and control subjects. One way analysis of covariance Kruskal-Wallis $\mathrm{H}$ test was used to investigate dose-response trends for the treatment with L-dopa and urine concentrations of Norsal derivatives. Changes in urine concentrations between $\mathrm{T} 1$ and $\mathrm{T} 2$ were evaluated using Wilcoxon matched pairs signed rank test. One way analysis of covariance with repeated measures design was performed for the urine levels of Norsal derivatives and the covariates age, gender as well as clinical parameters.

\section{RESULTS}

\section{Urine levels of Norsal derivatives}

In the control subjects, urine concentrations of norsal, NMNorsal and salsolinol were low (table 1). Equally low levels were found in three patients with early PD who did not receive L-dopa therapy. By contrast, PD patients under L-dopa treatment exhibited elevated urine concentrations of Norsal (NS), NMNorsal $(\mathrm{p}<0.05)$ and salsolinol $(\mathrm{p}<0.05)$ (table 1$)$. Sixteen of 44 patients under L-dopa treatment had urine concentrations of two or more Norsal derivatives that were higher than two standard deviations above the mean corresponding urine concentrations in controls.

When patients receiving L-dopa were divided into subgroups with low (<300 mg), medium (300-600 mg) and high $(>600 \mathrm{mg}$ ) daily dosage, a significant trend between the L-dopa dosage and the urine concentrations of salsolinol $(\mathrm{p}<0.05)$ and NMNorsal $(\mathrm{p}<0.01)$ was found in the KruskalWallis $\mathrm{H}$ test (table $\mathrm{l}$ ).

There was no association between increased urine concentrations of Norsal derivatives in the PD group and drugs other than L-dopa; we also found no association with the patients' age, gender, age at the onset of PD, disease duration, severity of the motor disability, or deficits in the cognitive performance assessed by the MMSE.

\section{Time course}

Between $\mathrm{T} 1$ and $\mathrm{T} 2$, urine concentrations of salsolinol decreased in $73 \%$ of the PD patients, NMNorsal levels declined in $65 \%$, and Norsal concentrations were reduced in $61 \%$. The difference between urine concentrations at the time points $\mathrm{T} 1$ and $\mathrm{T} 2$ was significant for salsolinol $(\mathrm{p}<0.01)$, NMNorsal $(p<0.05)$ and Norsal $(p<0.05)$ in the patient group, whereas no significant changes were found in controls (table 1).

Despite the decrease over time, urine levels of salsolinol (NS), Norsal $(p<0.05)$ and NMNorsal $(p<0.05)$ were still elevated at T2 in those PD patients who received L-dopa therapy compared with controls. In PD patients without L-dopa treatment, urine concentrations remained low (table 1).

\section{DISCUSSION}

Using a prospective, long term study design, we investigated the presence of Norsal derivatives in PD patients with regard to their drug treatment. We found a significant association between urine levels of Norsal derivatives and L-dopa treatment. A short term increase in salsolinol in the urine of PD patients following the intake of trial doses of L-dopa has been described previously. ${ }^{16}$ Our results show that urine levels of Norsal derivatives remain elevated continuously during L-dopa therapy. This L-dopa related, systemic increase in Norsal derivatives is dose dependent, with urine concentrations of salsolinol and NMNorsal being highest in PD patients receiving more than $300 \mathrm{mg}$ L-dopa daily. It is unlikely that the correlation between urine concentrations of Norsal derivatives and the L-dopa dosage is biased by disease progression and a subsequent rise in the drug dosage. The highest NMNorsal levels were measured in patients with less advanced disease as indicated by the UPDRS score. In accordance with a recent investigation of salsolinol concentrations in the blood plasma, ${ }^{9}$ urine levels of Norsal derivatives were not elevated in untreated patients with early PD; however the number of these patients was small. These results suggest that increased systemic concentrations of Norsal derivatives are the consequence of L-dopa treatment and cannot be attributed to alterations in the endogenous dopamine metabolism of patients with PD.

The ability of Norsal derivatives to pass the blood-brain barrier is limited. ${ }^{17}{ }^{18}$ Therefore, urine and blood levels most

Table 1 Urine concentrations of norsalsolinol, N-methyl-norsalsolinol, and salsolinol

\begin{tabular}{|c|c|c|c|c|c|c|}
\hline & \multicolumn{2}{|l|}{ Norsalsolinol } & \multicolumn{2}{|c|}{$N$-methyl-norsalsolinol } & \multicolumn{2}{|l|}{ Salsolinol } \\
\hline & $\mathrm{Tl}$ & T2 & $\mathrm{Tl}$ & T2 & T1 & T2 \\
\hline $\begin{array}{l}\text { Control subjects } \\
(n=14) \\
\text { PD patients }\end{array}$ & $\begin{array}{l}0.04(0.04) \\
(0-0.11)\end{array}$ & $\begin{array}{l}0.04(0.06) \\
(0-0.13)\end{array}$ & $\begin{array}{l}0.71(0.84) \\
(0-2.11)\end{array}$ & $\begin{array}{l}0.75(1.28) \\
(0-3.40)\end{array}$ & $\begin{array}{l}0.08(0.15) \\
(0-0.45)\end{array}$ & $\begin{array}{l}0.14(0.17) \\
(0-0.48)\end{array}$ \\
\hline $\begin{array}{l}\text { Untreated } \\
(n=3) \\
\text { Treated, total }(n=44 \\
\text { at T1, } 38 \text { at T2*) } \\
\text { Levodopa dosage }\end{array}$ & $\begin{array}{l}0.03(0.04) \\
(0-0.07) \\
0.51(1.24) \\
(0-5.93)\end{array}$ & $\begin{array}{l}0.05(0.01) \\
(0.03-0.06) \\
0.18(0.41) \\
(0-2.18)\end{array}$ & $\begin{array}{l}0.01(0.01) \\
(0-0.01) \\
3.31(4.87) \\
(0.14-18.27)\end{array}$ & $\begin{array}{l}\text { Not detected } \\
2.39(3.94) \\
(0-15.40)\end{array}$ & $\begin{array}{l}0.11(0.10) \\
(0.01-0.20) \\
1.04(1.22) \\
(0-6.47)\end{array}$ & $\begin{array}{l}0.30(0.16) \\
(0.14-0.46) \\
0.69(1.72) \\
(0-9.16)\end{array}$ \\
\hline $\begin{array}{l}<300 \mathrm{mg} / \text { day } \\
(\mathrm{n}=15) \\
300-600 \mathrm{mg} / \text { day } \\
(\mathrm{n}=24) \\
>600 \mathrm{mg} / \text { day } \\
(\mathrm{n}=5)\end{array}$ & $\begin{array}{l}0.45(1.16) \\
(0-4.75) \\
0.50(1.30) \\
(0-5.93) \\
0.55(0.99) \\
(0-2.31)\end{array}$ & & $\begin{array}{l}1.28(0.80) \\
(0.42-2.54) \\
4.08(5.92) \\
(0.14-18.27) \\
6.09(5.41) \\
(2.27-9.92)\end{array}$ & & $\begin{array}{l}0.63(0.66) \\
(0-2.16) \\
1.21(1.53) \\
(0-6.47) \\
1.30(0.68) \\
(0.39-1.92)\end{array}$ & \\
\hline
\end{tabular}

*Six patients died during the follow up period.

Data are given as mean (SD) (range) in $\mathrm{nmol} / \mathrm{ml}$ 
likely reflect the systemic synthesis of Norsal derivatives following the therapeutic administration of L-dopa and a subsequent rise in dopamine. ${ }^{19}$ Remarkably, the urine concentrations of Norsal derivatives declined significantly during the disease course. A similar decrease over time has been reported in a re-examination of $\mathrm{PD}$ patients with elevated CSF levels of $\mathrm{N}$-methylsalsolinol. ${ }^{20}$ Our results and the time course of $N$-methylsalsolinol in the $\mathrm{CSF}^{20}$ indicate a parallel decline of Norsal derivatives on both sides of the blood-brain barrier. We hypothesise that in PD patients, a genetic predisposition leads to alterations in the metabolism of endogenous dopamine in the nigrostriatal system and therapeutically administered L-dopa in the periphery, eventually resulting in the formation of Norsal derivatives, and that this altered dopamine metabolism is operating more effectively at the beginning of the disease.

Previous investigations have shown that Norsal derivatives are elevated in the brain or CSF of patients with PD regardless of their treatment with L-dopa. ${ }^{235}$ Consequently, Norsal derivatives in the CSF may indeed serve as biological markers of PD, whereas systemic levels of Norsal derivatives depend on the L-dopa treatment of patients. Systemically and in the CSF, the concentrations of Norsal derivatives decline over the disease course so that early determination is recommended.

\section{ACKNOWLEDGEMENTS}

We wish to thank K Schnackenberg and K Wiegers for their technical assistance.

\section{Authors' affiliations}

J Scholz, I Klingemann, A Moser, Neurochemistry Research Group, Department of Neurology, Medical University of Lübeck, Germany J Scholz, Neural Plasticity Research Group, Department of Anesthesia and Critical Care, Massachusetts General Hospital and Harvard Medical School, Charlestown, MA, USA

Competing interests: none declared

Correspondence to: Dr J Scholz, Neural Plasticity Research Group, Department of Anesthesia and Critical Care, Massachusetts General Hospital and Harvard Medical School, 149 13th Street, Room 4309, Charlestown, MA 02129, USA; scholz.joachim@mgh.harvard.edu

Received 14 January 2003

In revised form 8 August 2003

Accepted 2 September 2003

\section{REFERENCES}

1 Nagatsu T. Isoquinoline neurotoxins in the brain and Parkinson's disease. Neurosci Res 1997;29:99-111.

2 Niwa T, Takeda N, Yoshizumi H, et al. Presence of 2-methyl-6,7-dihydroxy1,2,3,4-tetrahydroisoquinoline and 1,2-dimethyl-6,7-dihydroxy-1,2,3,4tetrahydroisoquinoline, novel endogenous amines, in parkinsonian and normal human brains. Biochem Biophys Res Commun 1991;177:603-9.

3 Moser A, Kömpf D. Presence of methyl-6,7-dihydroxy-1,2,3,4tetrahydroisoquinolines, derivatives of the neurotoxin isoquinoline, in parkinsonian lumbar CSF. Life Sci 1992;50:1885-91.

4 Maruyama W, Narabayashi H, Dostert $P$, et al. Stereospecific occurrence of a parkinsonism-inducing catechol isoquinoline, $\mathrm{N}$-methyl(R)salsolinol, in the human intraventricular fluid. J Neural Transm 1996;103:1069-76.

5 Moser A, Scholz J, Nobbe F, et al. Presence of N-methyl-norsalsolinol in the CSF: correlations with dopamine metabolites of patients with Parkinson's disease. J Neurol Sci 1995;131:183-9.

6 Maruyama W, Abe T, Tohgi $\mathrm{H}$, et al. A dopaminergic neurotoxin, (R)-Nmethylsalsolinol increases in parkinsonian cerebrospinal fluid. Ann Neurol 1996:40:119-22.

7 Moser A, Siebecker F, Vieregge P, et al. Salsolinol, catecholamine metabolites, and visual hallucinations in I-DOPA treated patients with Parkinson's disease. J Neural Transm 1996;103:421-32.

8 Dostert P, Strolin Benedetti M, Della Vedova F, et al. Dopamine-derived tetrahydroisoquinolines and Parkinson's disease. Adv Neurol 1993;60:218-33.

9 Müller T, Sallström Baum S, Häussermann P, et al. Plasma levels of R- and S-salsolinol are not increased in "de novo" parkinsonian patients. J Neural Transm 1998; 105:239-46.

10 Müller T, Sallström Baum S, Häussermann P, et al. R- and S-salsolinol are not increased in cerebrospinal fluid of parkinsonian patients. J Neurol Sci 1999;164:158-62.

11 Ward CD, Gibb WR. Research diagnostic criteria for Parkinson's disease. Adv Neurol 1990;53:245-9.

12 Feest U, Kemper A, Nickel B, et al. Comparison of salsolinol excretion in alcoholics and nonalcoholic controls. Alcohol 1992;9:49-52.

13 Strolin Benedetti M, Dostert P, Carminati P. Influence of food intake on the enantiomeric composition of urinary salsolinol in man. J Neural Transm Gen Sect 1989;78:43-51.

14 Fahn S, Elton E, UPDRS Development Committee. Unified Parkinson's Disease Rating Scale. In: Fahn S, Marsden CD, Golstein M, Calne CD, eds. Recent developments in Parkinson's disease. Florham Park: Macmillan, 1992:153-63.

15 Folstein MF, Folstein SE, McHugh PR. Mini-Mental State: a practical method for grading the cognitive state of patients for the clinician. J Psychiatr Res 1975;12:189-98.

16 Dostert P, Strolin Benedetti M, Dordain G, et al. Enantiomeric composition of urinary salsolinol in parkinsonian patients after Madopar. J Neural Transm Park Dis Dement Sect 1989;1:269-78.

17 Origitano TC, Hannigan J, Collins MA. Rat brain salsolinol and blood-brain barrier. Brain Res 1981;224:446-51.

18 Thümen A, Behnecke A, Qadri F, et al. N-Methyl-norsalsolinol, a putative dopaminergic neurotoxin, passes through the blood-brain barrier in vivo. Neuroreport 2002;13:25-8.

19 Nutt JG, Woodward WR, Anderson JL. The effect of carbidopa on the pharmacokinetics of intravenously administered levodopa: the mechanism of action in the treatment of parkinsonism. Ann Neurol 1985;18:537-43.

20 Maruyama W, Abe T, Tohgi H, et al. An endogenous MPTP-like dopaminergic neurotoxin, $\mathrm{N}$-methyl(R)salsolinol, in the cerebrospinal fluid decreases with progression of Parkinson's disease. Neurosci Lett 1999;262:13-16. 